\title{
Novel Reflectometry Method Based on Time Reversal for Cable Aging Characterization
}

\author{
Lola El SAHMARANY*, Fabrice AUZANNEAU*, Pierre BONNET** \\ (*)Embedded Systems Reliability Laboratory, CEA, LIST, Nano-Innov, Essonne, France \\ ${ }^{* *}$ Institut Pascal, Blaise Pascal University, Clermont-Ferrand, Puy-de-Dôme, France \\ E-mail: lola.el-sahmarany@ cea.fr
}

\begin{abstract}
This paper investigates the effects of aging on electrical cable characteristics and proposes a new method for detecting and characterizing cable aging (i.e. homogeneous slow degradation) based on time reversal. In case of a global cable aging, the commonly used methods such as reflectometry provide non-relevant or inaccurate information. Through theoretical study and numerical simulations, the benefits of this new method called Time Reversal Reflectometry (TRR) are presented. TRR is experimentally shown to be successful for the detection and quantification of cable aging.
\end{abstract}

Index Terms-Aged cable, Transmission line, Reflectometry, Time Reversal, Skewness coefficient.

\section{INTRODUCTION}

Cables exist in all electrical systems, and are used to transmit power or data. The problem of cables aging has recently gained an increasing importance [1]-[5] and is identified as a critical safety factor in several application domains such as: transportation systems, industrial machinery, buildings, nuclear facilities, power distribution systems, etc. because a failure in any of these systems may cause injury to human beings.

Aging is described as a slow structural modification which gradually decreases the efficiency of an object, information or organism to provide its functions [6]. Therefore, this paper focuses on the detection and estimation of electrical cable aging.

The cable (insulators) properties are reversibly or irreversibly degraded by time, based on many factors such as: electric field, temperature, moisture, radiations, chemical corrosion, mechanical vibration, etc. The degradation presents unfavorable effects on electrical material that cause the appearance of failures which may lead to rupture and electric discharges of insulators.

Accordingly, knowledge and evaluation of cable aging are very important and can help to anticipate a timely maintenance for degraded cables. Therefore, a reliable method able to monitor cable status and provide reliable information about its degradation is needed.

In the literature several methods are used for testing cables, but reflectometry [7]-[12] is considered as the most reliable one. Reflectometry is well suited to detect and locate cable defects, but in case of an overall cable aging, it does not provide relevant information.
The research overcomes reflectometry's limitations by proposing a new approach based on time reversal [13], [14] applied to reflectometry's fundamental principle.

The feasibility, applicability, and accuracy of the proposed method are investigated by numerical simulation and experimental measurements. The simulation is done using a frequency model of cable in MATLAB ${ }^{\circledR}$ [8], [9]. For deeper investigation, the proposed method is applied experimentally on two identical healthy and aged cables for comparison purpose. This method proves to be beneficial, applicable and accurate when compared to standard reflectometry, as it permits to increase the detection and estimation efficiencies of aged cables.

The paper is organized as follows: section II briefly recalls reflectometry's principles, then section III introduces and proposes a theoretical model for the new methodology based on time reversal. Finally, the Time Reversal Reflectometry (TRR) advantages are shown in section IV by experimental measurements.

\section{STANDARD REFLECTOMETRY}

Standard reflectometry launches a pulse signal $v_{i}(t)$ down the cable, for example a Gaussian pulse as shown below.

$$
v_{i}(t)=A \cdot e^{-0.5\left(\frac{t}{a}\right)^{2}} \stackrel{f f t}{\Longrightarrow} V_{i}(f)=A \cdot \frac{1}{\sqrt{2 \cdot a}} e^{-0.5\left(\frac{f}{a}\right)^{2}}
$$

where, $A$ is the signal amplitude and $a$ is a scale factor which enables to adjust the temporal width (the Gaussian pulse bandwidth). $V_{i}(f)$ is the Fourier transform of $v_{i}(t)$ in (1). The wave propagates to the cable's far end, where it is reflected back and analyzed at the cable source. The reflected signal is given by [7]

$$
V_{R}(f)=\Gamma \cdot V_{i}(f) \cdot e^{-2 \gamma(f) d}
$$

where $\Gamma$ is the far end reflection coefficient (3), it is calculated as follows:

$$
\Gamma=\frac{Z_{L}-Z_{0}}{Z_{L}+Z_{0}}
$$

with $Z_{0}=\sqrt{\frac{R+j L \omega}{G+j C \omega}}$ is the characteristic impedance of the cable and $Z_{L}$ is the load impedance at the cable's far end. The reflection coefficient $\Gamma$ equals -1 for short circuit and 1 for open circuit. $\gamma$ is the propagation constant of the transmission line represented by attenuation $\alpha$ and phase $\beta$ constants in (4)

$$
\gamma(\omega)=\alpha+j \beta=\sqrt{(R+j L \omega)(G+j C \omega)}
$$




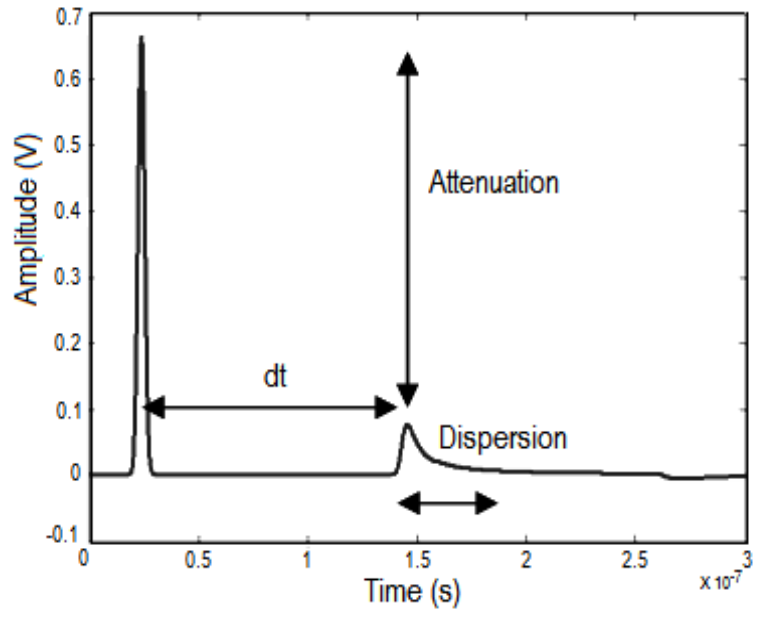

Fig. 1. Standard Reflectometry simulation results, showing the attenuation and the dispersion of the reflected signal in a $100 \mathrm{~m}$ long coaxial cable

The parameters $\omega, R, L, G$ and $C$ are the angular frequency in $\mathrm{rad} / \mathrm{sec}$, series resistance in $\Omega / m$, series inductance in $H / m$, shunt conductance in $\Omega^{-1} / m$, and shunt capacitance in $F / m$ respectively. In the current analysis, cable aging is represented by the modification of one or several electrical parameters $(R, L, G$, and $C$ ) values.

The cable's length $d$ is obtained by multiplying the time delay $d t$ (Fig. 1) by the propagation velocity $v_{p}$ :

$$
d=\frac{v_{p} \cdot d t}{2}
$$

The main problem in transmission lines is to choose the appropriate and precise value of the propagation velocity which depends on frequency and cable properties. It is necessary to accurately know the propagation velocity in the cable to calculate the cable's length and estimate its aging. Generally, the propagation velocity $v_{p}$ is calculated as follows:

$$
v_{p}=\frac{c}{\sqrt{\varepsilon_{r}}}
$$

where, $\varepsilon_{r}$ is the relative permittivity of the cable's dielectric insulator and $c$ is the speed of light.

Dielectric permittivity may change overtime due to various degradation processes such as thermal aging, corrosion, [15] and mechanical degradation [16]. This changing results in a significant variation of the propagation velocity [17] which is used to detect the aging of the cable .

However, it is difficult to know accurately the variation of $\varepsilon_{r}$ (therefore of the propagation velocity) and that increases the difficulty to evaluate the aging cable status. For example, in the experimental measurement presented on Fig.1, a Gaussian pulse (1) was sent into the cable under test (RG-58CU coaxial cable) by an arbitrary waveform generator (AWG).

Cable's attenuation reduces the amplitude of the input signal and its dispersion degrades the localization accuracy of this signal [18]-[21]. The cable dispersion distorts the propagated signal patterns which leads to a difficulty in measuring the time difference between incident and reflected signals as well as the precise value of the propagation velocity. Several techniques are proposed to overcome this problem but their application is expensive and complicated [11]. Therefore, a new approach based on time reversal applied to reflectometry is proposed in order to overcome these limitations.

\section{TIME REVERSAL REFLECTOMETRY}

This new method is based on the principles of time reversal and standard reflectometry method. It uses the fact that the reflectometry signal reflected by the cable's far end contains all the information about cable's status.

The proposed method uses the cable configuration described in section II, but instead of using a predefined signal (Gaussian pulse) like standard reflectometry method, it uses an adapted signal that allows the user to more precisely characterize the electrical parameters $(R L C G)$ modifications due to cable aging. The adapted signal will be insensitive to dispersion which distorts signals and decreases the ability of cable aging detection and estimation. The detection of cable aging using time reversal is resumed by the following process:

1) Inject a (symmetrical) pulse signal into a healthy cable.

2) If needed, truncate and shift in time, then normalize the reflected signal.

3) Apply time reversal, and then save as adapted signal.

4) Inject the adapted signal into the aged cable.

5) Process the reflected signal, calculate the skewness coefficient noted $S C$, and estimate the cable aging.

6) Loop steps 4 and 5 when needed.

In the following paragraph this process is analyzed and illustrated.

\section{A. Frequency domain mathematical analysis}

A mathematical model for the new method was developed in the frequency domain in order to demonstrate that the adapted signal after several round trips converges towards a signal which keeps the same shape after propagation. This study is summarized on Fig. 2, where $H(f)$ is the transfer function of the healthy cable, $H^{\prime}(f)$ for aged cable and $H^{*}(f)$ represents complex conjugate of $H(f)$ (time reversal in time domain) [14]. These different transfer functions is replaced with its values in the following mathematical study.

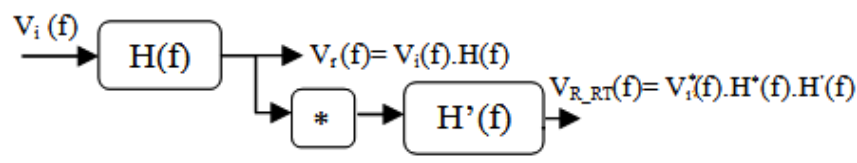

Fig. 2. Process of the new method in the frequency domain

When the signal $V_{i}(f)$ is injected down the cable, the reflected signal $V_{r}(f)$ can be calculated using equation (2). Time reversal is equivalent to complex conjugate in the frequency 
domain. Then, the so-called adapted signal is $V_{R}^{*}(f)$, denoting by "*" the complex conjugate, given by equation (7).

$$
V_{R}^{*}(f)=V_{i}^{*}(f) \cdot \Gamma^{*} \cdot e^{-2 \cdot \gamma^{*}(f) \cdot d}
$$

By injecting the adapted signal into the aged cable, the reflected signal of step 5 is obtained, which can be calculated by multiplying equation (7) by the transfer function of the aged cable as follows:

$$
V_{R-R T}(f)=V_{i}^{*}(f) \cdot \Gamma^{\prime} \Gamma^{*} \cdot e^{-2 \cdot d \cdot\left[\gamma^{\prime}(f)+\gamma^{*}(f)\right]}
$$

where, $\gamma^{\prime}(f)$ is the aged cable propagation constant and $\gamma^{*}(f)$ is the complex conjugate propagation constant of the healthy cable. If the cable under test is healthy then $\gamma^{\prime}(f)+$ $\gamma^{*}(f)$ is equal to twice the real part of $\gamma(f)$ and $V_{R-R T}(f)$ is real, meaning that the reflected signal of step 5 is symmetrical. Otherwise, as the cable parameters change over its age, the weak modifications of the aged cable's parameters induce an imaginary part in the frequency domain reflected signal, which implies that this signal is not symmetrical anymore in the time domain.

A mathematical demonstration of the asymmetry of the obtained signal is given below. For an aged cable the equation of the reflected signal is:

$$
V_{R-R T_{A}}(f)=V_{R-R T_{H}}(f) e^{2 \cdot d \cdot[\Delta \gamma(f)]}
$$

where, $\Delta \gamma(f)=\gamma^{\prime}(f)-\gamma(f)$.

$V_{R-R T_{A}}$ and $V_{R-R T_{H}}$ are the signals in the frequency domain for an aged and healthy cable respectively. Knowing that, the modulus of $2 d \Delta \gamma(f)$ is small compared to 1 because the modifications of electrical parameters are small. Using a Taylor expansion of equation (9), the equation for the aged cable is obtained as follows:

$$
V_{R-R T_{A}}(f)=V_{R-R T_{H}}(f)(1+2 d \Delta \gamma(f))
$$

where,

$$
\Delta \gamma(f)=\sum_{X \in\{R, L, C, G\}} \Delta X \frac{\partial \gamma(f)}{\partial X}
$$

The partial derivative of the equation (3) with respect to $R$ is:

$$
\frac{\partial \gamma(f)}{\partial R}=\frac{1}{2 Z_{0}}
$$

The same reasoning is applied for $G, L$ and $C$ respectively to obtain $\frac{Z_{0}}{2}, \frac{j w}{2 . Z_{0}}$, and $\frac{j w . Z_{0}}{2}$ respectively. Then, these four results of the partial derivative of equation (4) are added as follows:

$$
\Delta \gamma(f)=\frac{1}{2}\left(Z_{0}(\Delta G+j \omega \Delta C)+\frac{\Delta R+j \omega \Delta L}{Z_{0}}\right)
$$

Based on the aforementioned, a signal in the time domain is symmetrical when it is real in the frequency domain. So, the imaginary part of equation (13) provides the signal's asymmetry, which can be used to estimate the aging of the cable.

\section{B. Simulation results}

In order to investigate this method, numerical simulation is performed. The simulation is done by using a $R L C G$ frequency model of a cable using MATLAB ${ }^{\circledR}$.

In order to model an aged cable, different capacitance values are considered, each one represents a different age.

The effects of cable aging is detected by applying the process described in the section III, and a skewness coefficient noted $S C$ is computed. $S C$ is calculated by quantifying the signal's distortion on the left " $a$ " or the right side " $b$ " of its maximum as presented on Fig.3.

Then,

$$
S C=b / a
$$

A $S C$ value close to 1 means the cable under test is healthy. Otherwise, if the reflected signal is asymmetrical or $S C$ value is far from 1, this means the cable is aged and the value of $S C$ enables to quantify the aging.

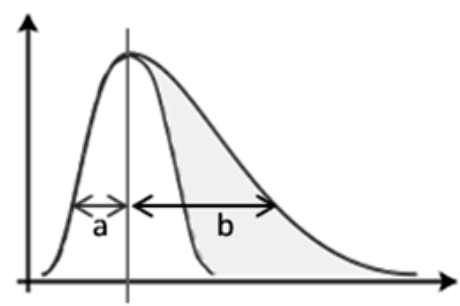

Fig. 3. Example of calculation of the skewness coefficient $S C$

Two (healthy and aged) 500 meter long transmission lines with $50 \Omega$ characteristic impedance, terminated by an open circuit at their far end, are used in this analysis. The two figures 4 and 5 are obtained after applying the fifth steps of the process described above for a healthy and an aged cable. Fig.4.(a) presents the symmetrical signal of step 1 injected into the $500 \mathrm{~m}$ healthy cable. After propagation, the cable's response presented in Fig.4.(b) is saved. Fig.4.(c) shows the result of the time reversal of the reflected signal saved in step 2 and called the adapted signal. This signal is injected into a cable under test to detect its aging. Fig.4.(d) shows the symmetry of the response of the healthy cable. On the contrary, figure 5 presents the response of an aged cable. So, the result of the figures 4.(d) and 5 show that the difference between the shapes of the signal is an indicator of the aging cable, as in the second figure (Fig.5) the signal is not symmetrical.

Then, a comparison by changing the per unit length capacitance value was performed. Fig. 6 shows the reflected signal from step 5 for the healthy cable (Capacitance is $C_{0}$ ) and for three simulated aged cables (Capacitance values of aged cables are $0.2,0.6$ and 1.2 times $C_{0}$ ).

Table I presents the calculated values of $S C$ and shows the effect of aging. When the cable is healthy, $S C$ is equal to 1 

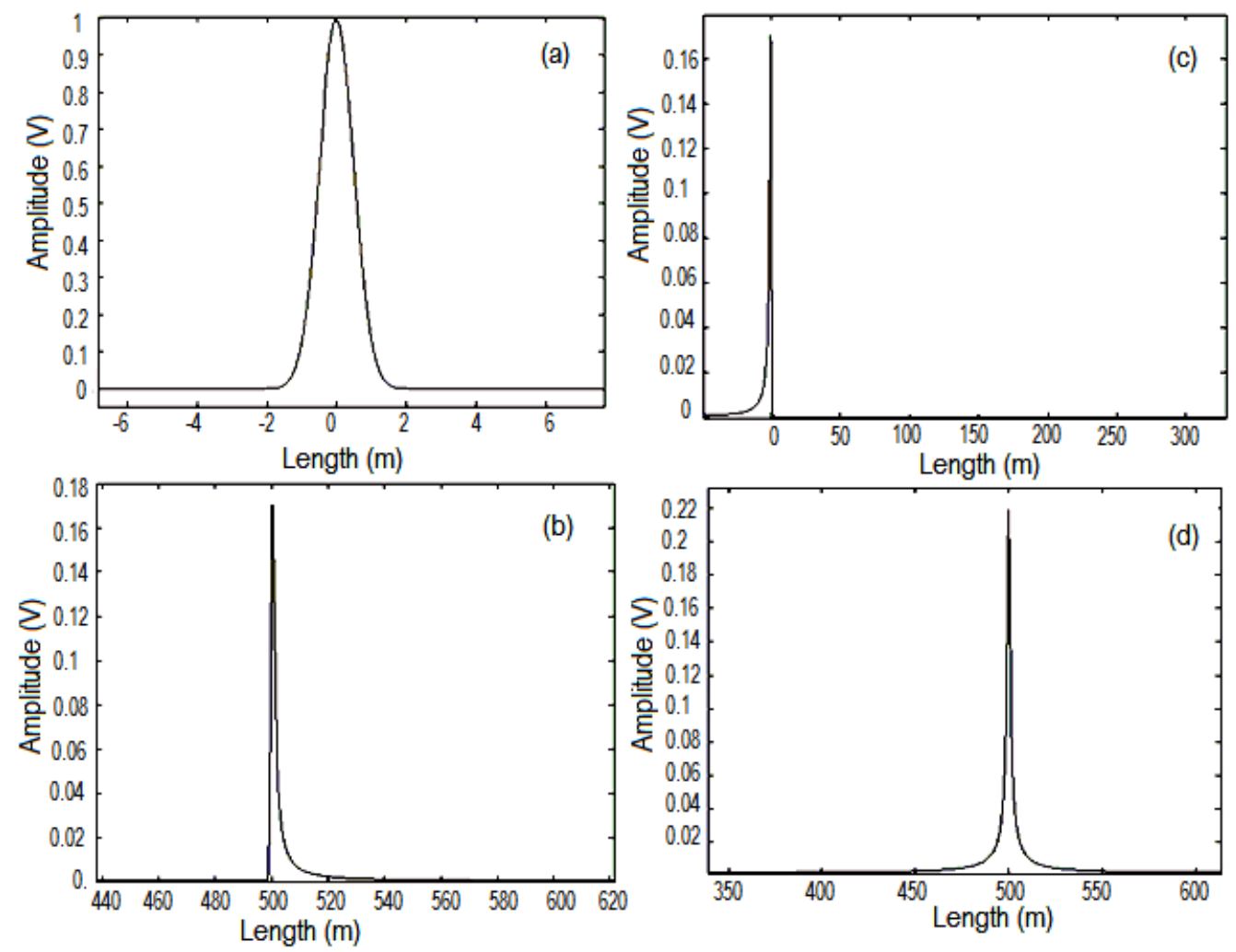

Fig. 4. Simulation results: (a) the injected symmetrical signal of step 1, (b) the reflected signal of step 2, (c) the time reversal of reflected signal to be injected in step 4, (d) the reflected signal of step 5, for a healthy cable

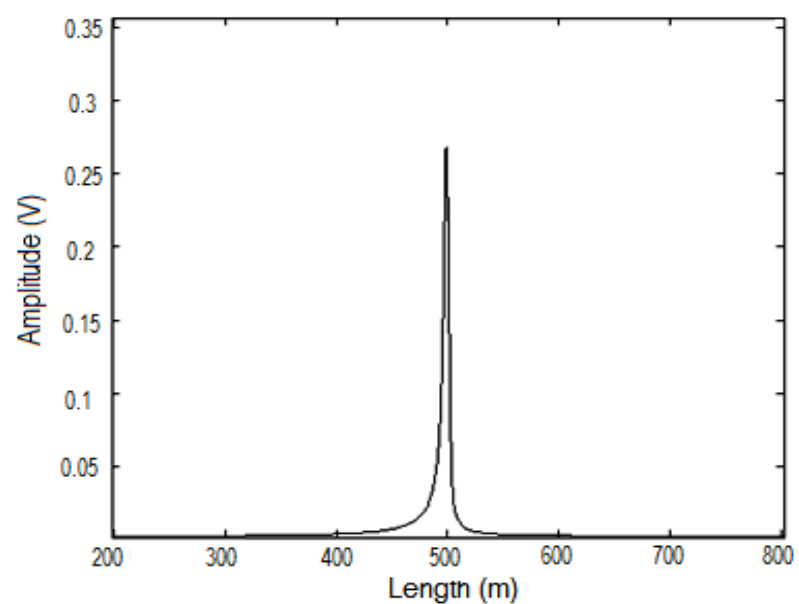

Fig. 5. The reflected signal of step 5 for an aged cable $\left(\left(0.2 * C_{0}\right)\right)$

and when it is aged $\left(0.2 * C_{0}\right) S C$ is down to 0.5127 .

In case the reference signal (adapted signal of step 3, used to detect the aging of the cable) of the healthy cable is lost, it is still possible to estimate the effects of aging. If we apply the

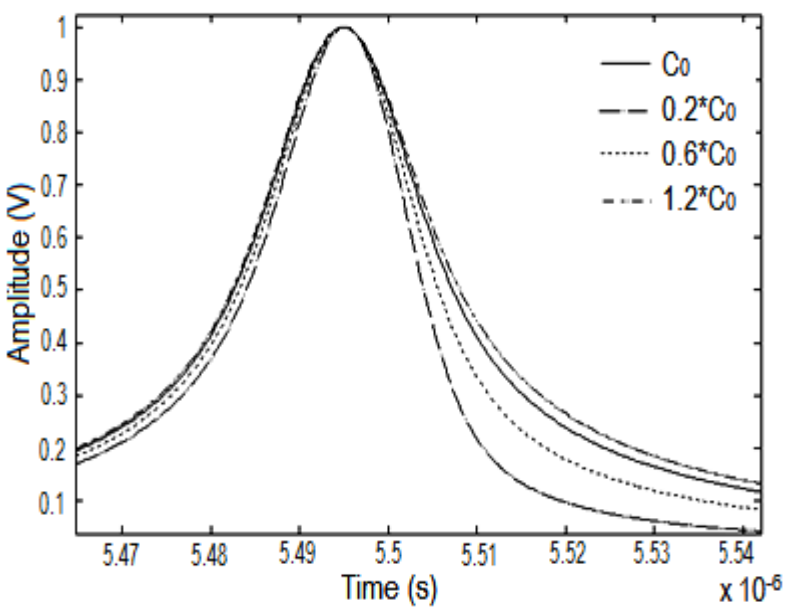

Fig. 6. The reflected signals of aged cable with different value of the capacitance

process from steps 1 to 4 to the healthy cable and to the aged cable, the signals of step 5 are different. Fig.7 compares the reflected signals of a healthy cable and an aged cable $\left(C_{0} * 0.2\right.$ and $C_{0} * 1.2$ ). The change in the width of the reflected signals 
TABLE I

VALUES OF SKEWNESS COEFFICIENT $S C$ FOR DIFFERENT AGED CABLES

\begin{tabular}{|c|c|c|c|c|}
\hline Simulations & $1.2 * C_{0}$ & $C_{0}$ & $0.6 * C_{0}$ & $0.2 * C_{0}$ \\
\hline Skewness Coefficient & 1.09 & 1 & 0.7943 & 0.5127 \\
\hline
\end{tabular}

shows the effects of aging, and can easily be quantified.

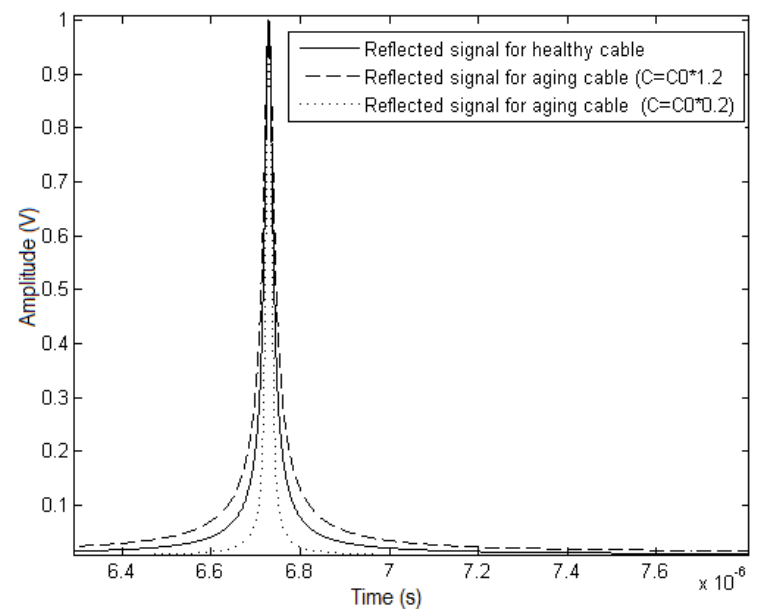

Fig. 7. Comparison between a healthy cable and aged cable using the TRR in case of lost of the reference

\section{EXPERIMENTAL SET UP}

Thermal aging represents an important stress which can greatly affect the electrical insulation properties of a cable and may limit its effective service life by thermal breakdown mechanisms.

Therefore, several experimental measurements were done to evaluate the TRR method results.

Thermal aging experiment is performed on a $100 \mathrm{~m}$ long coaxial cable during two months and a half at $105^{\circ} \mathrm{C}$ temperature (Fig.8). The detection of cable aging using our time reversal approach is performed like in the simulation section by following these steps:

1) Inject a Gaussian pulse signal into the healthy cable using an Arbitrary Waveform Generator (AWG).

2) Measure the reflected signal with a digital oscilloscope.

3) Using MATLAB ${ }^{\circledR}$, the reflected signal is truncated, shifted, normalized and time reversal is applied to get the adapted signal, which is saved.

4) During aging tests, the computer running Labview controls the AWG to periodically inject the adapted signal from step 3 into the cable.

5) Process reflected signal, calculate $S C$, and estimate aging.

Before the aging experiment began, a reference was saved (response of the open ended cable) to do a comparison with the measurements during aging. Every five days, the response of the open ended cable is measured. The obtained results show that the thermal aging affects considerably the electrical and mechanical properties of the material. It was also noted that the material loses rapidly its properties with higher temperature.

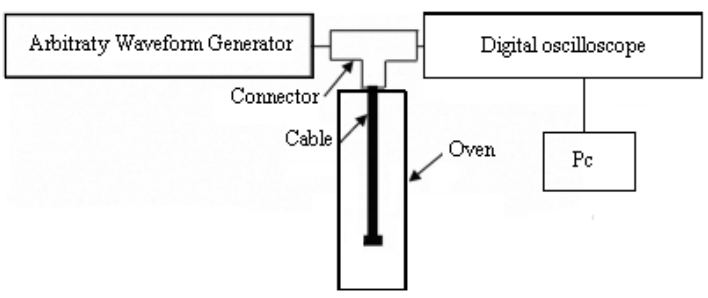

Fig. 8. Block diagram of the experimental setup

Fig.9 shows the variation of the reflected signals from step 5 for four different aging values. The measurement number 1 is for the healthy cable. It is noticeable that the signal is symmetrical because $S C$ is equal to 1 . The measurements two, three, and four are saved after one month and half, two months, and two months and ten days respectively. Table II shows the effect of aging during the experimental study via the variation of the skewness coefficient $S C$ value. It was noted that the increasing of $S C$ with time led the reflected signal to lose its symmetry.

TABLE II

VALUES OF THE SKEWNESS COEFFICIENT DURING AGING

\begin{tabular}{|c|c|c|c|c|}
\hline Measure & 1 & 2 & 3 & 4 \\
\hline Skewness Coefficient & 1 & 1.2809 & 1.2966 & 1.3154 \\
\hline
\end{tabular}

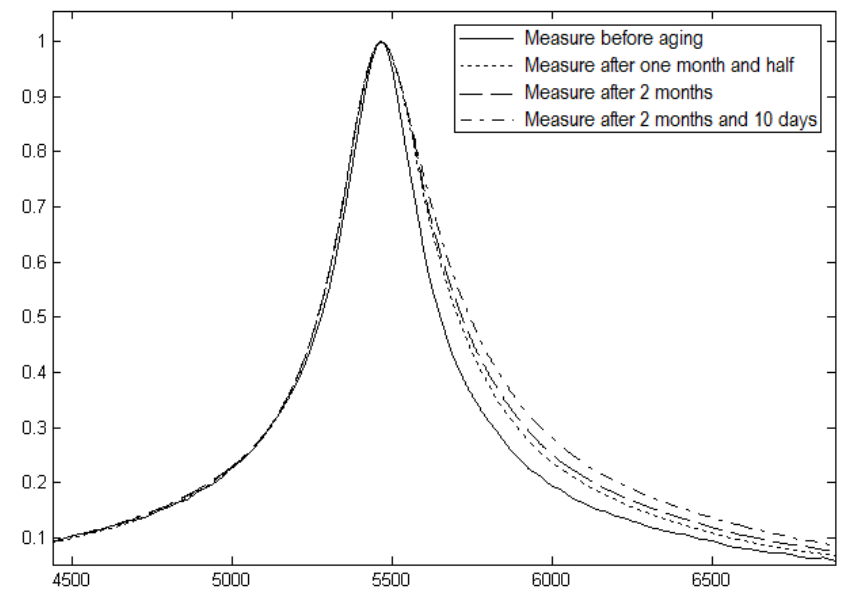

Fig. 9. The reflected signals during aging

\section{CONCLusion}

This paper presented a new method for precise cable aging detection and estimation based on time reversal and standard reflectometry method. Time Reversal Reflectometry (TRR) shows great ability for detecting and assessing aging degree. The TRR was demonstrated by performing a mathematical 
analysis on the non symmetrical reflected signal of an aged cable.

The advantages of the Time Reversal Reflectometry over standard Reflectometry have been shown also by experimental and simulation results. During accelerated aging test for a $100 \mathrm{~m}$ coxial cable, TRR was employed to monitor the state of an aging cable and it shows good results. Even a small variation of $S C$ represents an index of aging.

It should be noted that, using only standard reflectometry result to monitor the variation of cable permittivity during the aging process provides inaccurate results, mainly because the variation of the permittivity value influences the propagation velocity. So, the proposed method avoids this problem by presenting a simple and more accurate technique to estimate the cable aging.

In addition, the TRR method can also work in case a non symmetrical signal is injected of step 1 . The $S C$ coefficient of the signal of step 5 is equal to the $S C$ coefficient of the initial signal for healthy cable and will be different for an aged cable.

Finally, the knowledge and evaluation of cable aging can help to anticipate timely maintenance of degraded cables. The health of the cables and the safety of an entire electrical system can be ensured by using the developed method which gives the ability to continuously monitor the cable functioning status.

\section{REFERENCES}

[1] I. Standard-1064-1991:, "Guide for multifactor stress functional testing of electrical insulation systems," 1991.

[2] S. Dalal and R. Gorur, "Aging of distribution cables in service and its simulation in the laboratory," IEEE Trans. Dielectr. Electr. Insulation, vol. 12, no. 1, 2005.

[3] K. Anandakumaran, W. Seidl, and P. Castaldo, "Condition assesment of cable insulation systems in operating nuclear power plants," IEEE Trans. Dielectr. Electr. Insul., vol. 6, no. 3, 1999.

[4] S. P. Cygan and J. R. Laghari, "Effects of multistress aging (radiation, thermal, electrical) on polypropylene," IEEE Trans. Nuclear Sci., vol. 38, no. 3, 1991.

[5] C. Furse and R. Haupt, "Down to the wire: the hidden hazard of aging aircraft wiring," IEEE Spectrum, vol. 38, 2001.

[6] N. Lahoud, "Modélisation du vieillissement des isolants organiques sous contrainte électrique. application la fiabilité des matériaux," Ph.D. dissertation, University of Toulouse III, 2009.

[7] F. Auzanneau, M. Olivas, and N. Ravot, "A simple and accurate model for wire diagnosis using reectometry," PIERS Proceedings, Prague, Czech Republic, vol. 22, no. 1, August 2007.

[8] M. Smail, L. Pichon, M.Olivas, F. Auzanneau, and M.Lambert, "Detection of defects in wiring networks using time domain reflectometry," Magnetics, IEEE Transactions on, vol. 46, Issue: 8, Aug. 2010.

[9] N. Ravot, F. Auzanneau, Y. Bonhomme, M. Olivas, and F. Bouillault, "Distributed reflectometry-based diagnosis for complex wired networks," EMC: Safety, Reliability and Security of Communication and Transportation Systems, Paris, june 2007.

[10] C. Furse, Y. C. Chung, C. Lo, and P. Pendayala, "A critical comparison of reflectometry methods for location of wiring faults," Smart Structures and Systems, vol. 2, no. 1, 2006.

[11] Q. Shi, U. Troeltsch, and O. Kanoun, "Detection and localization of cable faults by time and frequency domain measurements," 7th International Multi-Conference on Systems, Signals and Devices, vol. 2, no. 1,2010
[12] J. Wang, P. Stone, J. Shin, and R. Dougal, "Application of joint timefrequency domain reflectometry for electric power cable diagnosis," IET signal processing, USA, 2009.

[13] M. Fink, "Time reversal of ultrasonic fields part 1: basic principles," IEEE Transactions on Ultrasonics, Ferroelectrics, and Frequency Control, vol. 39, No. 5, 555 566, Sep. 1992.

[14] A. Abboud L. and L. Pichon, "Utilization of matched pulses to improve fault detection in wire networks," The 9th International Conference on ITS Telecommunications, Lille (France), Oct. 2009.

[15] W. Liu, R. Hunsperger, K. Folliard, M. Chajes, J. Barot, D. Jhaveri, and E. Kunz, "Detection and characterization of corrosion of bridge cables by time domain reflectometry," Nondestructive evaluation of aging aircraft, airports, and aerospace hardware, Newport Beach CA, USA, vol. 3587, pp. 28-39, 1999.

[16] L. Li, N. Bowler, M. R. Kessler, , and S. H. Yoon, "Dielectric response of ptfe and etfe wiring insulation to thermal exposure," IEEE Transactions on Dielectrics and Electrical Insulation, vol. 17, No. 4, August 2010.

[17] T. E. D. E. Sayed, M. Gilany, M. M. A. Aziz, and D. khalil Ibrahim, "A wavelet-based fault location technique for aged power cables," IEEE Power Engineering Society General Meeting, vol. 2485 - 2491, No. 4, 2005.

[18] J. Kojima, "Optimization of pulse width for electric time domain reflectometry for fault point localization of power feeding lines of optical submarine cables," Workshop on Scientific Use of Submarine Cables and Related Technologies (SSC),Underwater Technology (UT), 2011.

[19] L. Cohen, "Wave propagation with dispersion and damping," Proc. SPIE, Denver, USA, vol. 001.5559, pp. 201220, 2004.

[20] C. S. Chen and L. E. Roemer, "Attenuation and dispersion compensation via cepstral processing," IEEE transactions on acoustics, speech, and signal processing, vol. Asp-29, no. 5, October 1981.

[21] "Time domain reflectometry theory," Application Note 1304-2, Agilent Technologies, www.agilent.com, Aug. 2002. 